PROCEEDINGS OF THE

AMERICAN MATHEMATICAL SOCIETY

Volume 126, Number 11, November 1998, Pages 3223-3226

S $0002-9939(98) 04797-2$

\title{
EVERY GROUP HAS A TERMINATING TRANSFINITE AUTOMORPHISM TOWER
}

\author{
JOEL DAVID HAMKINS
}

(Communicated by Ronald M. Solomon)

\begin{abstract}
Iteratively taking the automorphism group of any group leads, transfinitely, to a fixed point.
\end{abstract}

The automorphism tower of a group is obtained by computing its automorphism group, the automorphism group of that group, and so on, iterating transfinitely. Each group maps canonically into the next using inner automorphisms, and so at limit stages one can take a direct limit and continue the iteration:

$$
G \rightarrow \operatorname{Aut}(G) \rightarrow \operatorname{Aut}(\operatorname{Aut}(G)) \rightarrow \cdots \rightarrow G_{\omega} \rightarrow G_{\omega+1} \rightarrow \cdots \rightarrow G_{\alpha} \rightarrow \cdots .
$$

The tower is said to terminate if a fixed point is reached, that is, if a group is reached which is isomorphic to its automorphism group by the natural map. This occurs if a complete group is reached, one which is centerless and has only inner automorphisms.

In the special case that the initial group is centerless, matters simplify considerably: in this case all the groups appearing in the tower are centerless (see Hulse [1970]), and, consequently, all the natural maps are injective. The tower can therefore be viewed as building upwards to larger and larger groups; the question is whether this building process ever stops. ${ }^{1}$ Wielandt [1939] proved the classical result that the automorphism tower of any centerless finite group terminates in finitely many steps. Rae and Roseblade [1970] proved that the automorphism tower of any centerless Cernikov group terminates in finitely many steps. Hulse [1970] proved that the automorphism tower of any centerless polycyclic group terminates in countably many steps (but not necessarily after just $\omega$ many steps). Solving the problem for centerless groups, Simon Thomas [1985] proved that the automorphism tower of any centerless group eventually terminates. In fact, the automorphism tower of a centerless group $G$ terminates in fewer than $\left(2^{|G|}\right)^{+}$many steps. In the general case, however, the question remained open whether every group has a terminating automorphism tower. This is settled by the following theorem.

Received by the editors April 9, 1997.

1991 Mathematics Subject Classification. Primary 20E36, 20F28.

The author's research has been supported in part by a grant from the PSC-CUNY Research Foundation. He would like to thank both Daniel Seabold and Daniel Velleman for pointing out a simplification in the proof.

${ }^{1}$ In Hulse [1970], Rae and Roseblade [1970], and Thomas [1985], the tower is only defined in this special case; but the definition I give here works perfectly well whether or not the group $G$ is centerless. Of course, when there is a center, one has homomorphisms rather than embeddings.

(C)1998 American Mathematical Society 
Main Theorem. Every group has a terminating automorphism tower.

Proof. Suppose $G$ is a group. The following transfinite recursion defines the automorphism tower of $G$ :

$$
\begin{aligned}
G_{0} & =G \\
G_{\alpha+1} & =\operatorname{Aut}\left(G_{\alpha}\right), \quad \text { where } \pi_{\alpha, \alpha+1}: G_{\alpha} \rightarrow G_{\alpha+1} \text { is the natural map, } \\
G_{\lambda} & =\operatorname{dir} \lim _{\alpha<\lambda} G_{\alpha}, \quad \text { if } \lambda \text { is a limit ordinal. }
\end{aligned}
$$

When $\alpha<\beta$ one obtains the map $\pi_{\alpha, \beta}: G_{\alpha} \rightarrow G_{\beta}$ by composing the canonical maps at each step, and these are the maps used to compute the direct limit at limit stages. Thus, when $\lambda$ is a limit ordinal, every element of $G_{\lambda}$ is of the form $\pi_{\alpha, \lambda}(g)$ for some $\alpha<\lambda$ and some $g \in G_{\alpha}$.

Since Simon Thomas [1985] has proved that every centerless group has a terminating automorphism tower, it suffices to show that there is an ordinal $\gamma$ such that $G_{\gamma}$ has a trivial center. For each ordinal $\alpha$, let $H_{\alpha}=\left\{g \in G_{\alpha} \mid \exists \beta \pi_{\alpha, \beta}(g)=1\right\}$. For every $g \in H_{\alpha}$ there is some least $\beta_{g}>\alpha$ such that $\pi_{\alpha, \beta_{g}}(g)=1$. Let $f(\alpha)=\sup _{g \in H_{\alpha}} \beta_{g}$. It is easy to check that if $\alpha<\beta$, then $\alpha<f(\alpha) \leq f(\beta)$. Iterating the function, define $\gamma_{0}=0$ and $\gamma_{n+1}=f\left(\gamma_{n}\right)$. This produces a strictly increasing $\omega$-sequence of ordinals whose supremum $\gamma=\sup \left\{\gamma_{n} \mid n \in \omega\right\}$ is a limit ordinal which is closed under $f$. That is, $f(\alpha)<\gamma$ for every $\alpha<\gamma$. I claim that $G_{\gamma}$ has a trivial center. To see this, suppose $g$ is in the center of $G_{\gamma}$. Thus, $\pi_{\gamma, \gamma+1}(g)=1$. Moreover, since $\gamma$ is a limit ordinal, there is $\alpha<\gamma$ and $h \in G_{\alpha}$ such that $g=\pi_{\alpha, \gamma}(h)$. Combining these facts, observe that

$$
\pi_{\alpha, \gamma+1}(h)=\pi_{\gamma, \gamma+1}\left(\pi_{\alpha, \gamma}(h)\right)=\pi_{\gamma, \gamma+1}(g)=1 .
$$

Consequently, $\pi_{\alpha, f(\alpha)}(h)=1$. Since $f(\alpha)<\gamma$, it follows that

$$
g=\pi_{\alpha, \gamma}(h)=\pi_{f(\alpha), \gamma}\left(\pi_{\alpha, f(\alpha)}(h)\right)=\pi_{f(\alpha), \gamma}(1)=1,
$$

as desired.

The proof does not reveal exactly how long the automorphism tower takes to stabilize, since it is not clear how large $f(\alpha)$ can be. Nevertheless, something more can be said. Certainly the automorphism tower of $G$ terminates well before the next inaccessible cardinal above $|G|$. More generally, if $\kappa>\omega$ is regular and $\left|G_{\alpha}\right|<\kappa$ whenever $\alpha<\kappa$, then I claim the centerless groups will appear before $\kappa$. To see this, let $H_{\alpha}=\left\{g \in G_{\alpha} \mid \exists \beta_{g}<\kappa \pi_{\alpha, \beta_{g}}(g)=1\right\}$ and define $f: \kappa \rightarrow \kappa$ by $f(\alpha)=\sup _{g \in H_{\alpha}} \beta_{g}$; if $\gamma<\kappa$ is closed under $f$, then it follows as in the main theorem that $G_{\gamma}$ has no center, as desired. In this case the tower therefore terminates in fewer than $\left(2^{\left|G_{\gamma}\right|}\right)^{+}$many additional steps. If it happens that $\left|G_{\gamma}\right|^{+}<\kappa$, one can adapt Thomas [1998] argument using Fodor's lemma to prove that the tower terminates actually in fewer than $\kappa$ many steps. The point is that one can find a bound on the height of the tower by bounding the rate of growth of the groups in the tower.

Thomas [1985] provides his explicit bound in the case of centerless $G$ in precisely this way. He proves that if $G$ is centerless and $\kappa=\left(2^{|G|}\right)^{+}$, then $\left|G_{\alpha}\right|<\kappa$ for all $\alpha<\kappa$. The analogous result, unfortunately, does not hold for groups with nontrivial centers. This is illustrated by the following example, provided by the anonymous referee of this paper: 
Example. There exists a countable group $G$ such that

$$
\mid \text { Aut } G \mid=2^{\omega} \quad \text { and } \quad|\operatorname{Aut}(\operatorname{Aut} G)|=2^{2^{\omega}} \text {. }
$$

Proof. For each prime $p$, let $\mathbf{Z}[1 / p]=\left\{m / p^{n} \mid m \in \mathbf{Z}, n \in \mathbf{N}\right\}$ be the additive group of $p$-adic rationals and let $G=\bigoplus_{p} \mathbf{Z}[1 / p]$ be the direct sum of these groups. An element $g \in G$ is divisible by $p^{n}$ for all $n \in \mathbf{N}$ iff $g \in \mathbf{Z}[1 / p]$. Hence, if $\pi \in$ Aut $G$, then $\pi[\mathbf{Z}[1 / p]]=\mathbf{Z}[1 / p]$ for each prime $p$. It is easy to see that any automorphism of $\mathbf{Z}[1 / p]$ is simply multiplication by an element $u \in U_{p}=\left\{ \pm p^{n} \mid n \in \mathbf{Z}\right\}$, the group of multiplicative units of the ring of $p$-adic rationals. Thus, Aut $G \cong \Pi_{p} U_{p}$; and so $\mid$ Aut $G \mid=2^{\omega}$.

Next note that $U_{p} \cong \mathbf{Z} \times C_{2}$ for each prime $p$. Thus Aut $G \cong P \times V$, where $P$ is the direct product of countably many copies of $\mathbf{Z}$ and $V$ is the direct product of countably many copies of $C_{2}$. Since each nonzero element of $V$ has order 2 , it follows that $V$ is isomorphic to a direct sum of $|V|$ copies of $C_{2}$. Thus we can identify $V$ with a vector space of dimension $2^{\omega}$ over the field of two elements. Hence,

$$
\operatorname{Aut}(\text { Aut } G) \cong \text { Aut } P \times \text { Aut } V=\text { Aut } P \times G L(V),
$$

where $G L(V)$ is the general linear group on the vector space $V$. Since $|G L(V)|=$ $2^{2^{\omega}}$, it follows that $|\operatorname{Aut}(\operatorname{Aut} G)|=2^{2^{\omega}}$.

Enriqueta Rodríguez-Carrington has observed that the natural modification of my argument shows that the derivation tower of every Lie algebra eventually leads to a centerless Lie algebra. Since Simon Thomas [1985] proved that the derivation tower of every centerless Lie algebra must eventually terminate, it follows that the derivation tower of any Lie algebra must eventually terminate.

One might hope, since every step of the automorphism tower kills the center of the previous group, that $G_{\omega}$ is always centerless; but this is not so. The dihedral group with eight elements has a center of size two, but is isomorphic to its own automorphism group (there is an outer automorphism which swaps $a$ and $b$ in the presentation $\left.\left\langle a, b \mid a^{2}=1, b^{2}=1,(a b)^{4}=1\right\rangle\right)$. The group at stage $\omega$ is just the two element group, which still has a center, and so this tower survives until $\omega+1$. Simon Thomas has constructed examples showing that for every natural number $n$ there are finite groups whose tower has height $\omega+n$, but these also become centerless at stage $\omega+1$. Perhaps our attention should focus, for an arbitrary group $G$, on the least ordinal stage $\gamma$ such that $G_{\gamma}$ is centerless. The main point, then, is to find an explicit bound on how large $\gamma$ can be in comparison with $|G|$.

To my knowledge, the following questions remain open: For which ordinals $\gamma$ is there a group whose tower becomes centerless in exactly $\gamma$ many steps? Is there a countable group with an uncountable automorphism tower? Is there a finite group with an uncountable automorphism tower? Is there a finite group $G$ such that $G_{\omega}$ is infinite?

\section{REFERENCES}

[1997] Joel David Hamkins and Simon Thomas, Changing the heights of automorphism towers, submitted to the Annals of Pure and Applied Logic.

[1970] J. A. Hulse, Automorphism towers of polycyclic groups, Journal of Algebra, 16, 1970, 347-398. MR 42:1888

[1970] Andrew Rae and James E. Roseblade, Automorphism Towers of Extremal Groups, Math. Z., 70-75, 1970, 117. MR 43:2069

[1985] Simon Thomas, The automorphism tower problem, Proceedings of the American Mathematical Society, 95, 1985, 166-168. MR 86k:20028 
[1998] Simon Thomas, The automorphism tower problem II, to appear in Israel J. Math.

[1939] H. Wielandt, Eine Verallgemeinerung der invarianten Untergruppen, Math. Z., 45, 1939, 209-244.

Department of Mathematics, City University of New York, College of Staten IsLANd, Staten Island, New York 10314

E-mail address: hamkins@integral.math.csi.cuny.edu

$U R L$ : www.library.csi.cuny.edu/users/hamkins/ 